\title{
Louis-André Gosse et la médecine pénitentiaire
}

Par Cléopâtre Montandon

Parler de la médecine pénitentiaire au $\mathrm{XIX}^{\mathrm{e}}$ siècle à Genève c'est s'interroger sur l'institution carcérale en général. En effet la prison n'est pas la seule stratégie qu'une société peut adopter face aux actes delictueux. Il a toujours existé dans le temps et dans l'espace des variations de la réaction sociale vis-à-vis des comportements qui transgressent les normes établies. De nombreux spécialistes dans le domaine de la criminologie sont d'avis que la réaction punitive, dans le sens d'une soumission à une peine carcérale, cette peine ayant une valeur en soi, n'a pris de l'importance que dans les temps modernes. Parmi les différentes techniques d'application de la peine on trouve la torture physique, la dégradation sociale, les sanctions économiques, l'extermination, l'exil, et l'incarcération. Si la réaction aux illégalismes est universelle, la forme de cette réaction est variable. Aucune technique punitive n'est caractéristique d'une seule époque ou d'un seul pays, mais l'accent est mis à certaines périodes historiques sur l'une ou l'autre de ces techniques. Ainsi, dans les sociétés anciennes ou dans les sociétés dites "primitives» l'emprisonnement était rarement prescrit comme l'exécution généralisée d'une sanction juridique. L'incarcération était un moyen de faire pression pour le paiement d'une dette ou s'avérait nécessaire pendant l'instruction; elle était également pratiquée par l'Eglise; ce n'était pas une sanction généralisée.

Cependant, à partir du XVI ${ }^{\mathrm{e}}$ siècle en Angleterre et peu après en Europe continentale, on constate l'apparition de maisons de correction, d'institutions de contrôle parapénales, où on enferme les vagabonds, les mendiants, les individus capables mais refusant de travailler.

L'importance attribuée à la peine, à la punition, n'est pas sans rapport avec le développement de théories sur l'étiologie de la criminalité. Les théoriciens du $\mathrm{XVIII}^{\mathrm{e}}$ siècle toutefois n'étaient pas persuadés de l'efficacité de l'enfermement comme peine; il était déjà connu que l'enfermement non seulement empêche le pouvoir judiciaire de contrôler l'application des peines mais a un effet néfaste sur les individus, la prison étant considérée comme une école du crime, un lieu qui attire certains délinquants ou alors qui les rend, en les stigmatisant, criminels à vie.

Cependant, il y eut au XVIII ${ }^{\mathrm{e}}$ siècle une convergence de deux facteurs principaux qui tout en étant en contradiction aboutirent à l'émergence d'une nouvelle conception pénale axée sur la réforme par la pénitence, sur la régénération en prison: d'une part l'emprisonnement se généralisait à cause de l'intolérance grandissante de la bourgeoisie face aux individus qui perturbaient l'ordre social 
établi, d'autre part apparaissait une dénonciation des conditions de l'enfermement liée à une conception humanitaire de l'homme et de la discipline, souvent dans le cadre de mouvements de réforme des prisons.

Une nouvelle théorie pénale s'est créée, qui, une fois instituée, a contribué par un effet de rétroaction à renforcer l'institution carcérale. Ainsi, en même temps que s'élabore le système pénitentiaire, se construit un discours pénitentiaire, un discours réformateur qui tout en critiquant le système contribue à le populariser. A Genève, comme ce fut le cas dans le reste de l'Europe et aux Etats-Unis, ce discours était produit par des juristes, des politiciens, des médecins. On peut se demander pourquoi des médecins ? En premier lieu on les appelait parfois pour soigner des prisonniers, même si ce n'était que pour les rendre plus à même d'apprécier leur punition. En deuxième lieu la prison était de plus en plus considérée comme le lieu où on doit guérir. Troisièmement, très vite des rapprochements furent établis entre maladie mentale et criminalité. Un intérêt pour les causes biologiques de la criminalité commençait à poindre. Il est intéressant de noter sur ce dernier point que les premières tentatives d'étude scientifique du crime étaient sociologiques plutôt que biologiques. Quételet, le mathématicien belge, et Guerry, un avocat français, avaient tenté depuis 1830, en analysant les statistiques nationales françaises sur la criminalité, d'étudier les variations du taux de criminalité selon des facteurs sociaux. Mais cette approche ne fut pas accueillie avec enthousiasme à une époque où on voulait surtout tenir tranquilles les «classes dangereuses $\gg{ }^{1}$ Le déterminisme biologique était plus acceptable que le positivisme sociologique car il soulignait l'importance des facteurs innés à l'individu et non pas le dysfonctionnement social. Ce fut Lombroso qui porta à l'absurde le déterminisme biologique en 1876 avec L'Uomo Delinquente.

Dans la réforme et la médicalisation du discours pénitentiaire, Louis-André Gosse, dont les principaux écrits se situent entre 1838 et 1864, occupe selon nous, même s'il n'est pas cité dans les ouvrages criminologiques, une place qui mérite notre attention. Son discours, caractéristique de l'école scientifique genevoise, un joyau de prudence helvétique, analyse avant Lombroso et de manière beaucoup plus subtile les facteurs biologiques qu'il considère comme ayant une influence sur la criminalité. Son cadre théorique, malgré certaines affirmations qui peuvent paraître naïves aujourd'hui, est en quelque sorte beaucoup plus près de celui d'auteurs contemporains, qui mettent en évidence l'interaction entre facteurs génétiques et environnementaux.

Ayant situé de manière extrêmement condensée les circonstances dans lesquelles le discours pénitentiaire s'est créé, nous aborderons après quelques brèves indications biographiques, l'analyse des écrits de Louis-André Gosse et plus parti- 
culièrement de son ouvrage, Examen médical et philosophique du système pénitentiaire $^{2}$.

Louis-André Gosse est né à Genève en 1791, dans une famille où l'on vénérait la science. Son grand-père était libraire et son père, physicien et pharmacien, dans l'officine duquel se donnaient rendez-vous les savants genevois de l'époque, était également membre de plusieurs sociétés scientifiques et avait créé en 1815 la Société Helvétique des Sciences Naturelles. C'était un homme «passionnément épris de la science et de ses applications pratiques, surtout lorsqu'il les croyait susceptibles de contribuer au soulagement des misères humaines ». ${ }^{3}$ Henri-Albert Gosse envoya son fils Louis-André aux frères Moraves de Neuwied pour sa première éducation, car les idées religieuses et morales de cette institution lui plaisaient, notamment le principe de sacrifier toujours l'intérêt particulier à l'intérêt général. Louis-André, devenu médecin en 1816 après des études à Paris, déploya un zèle extraordinaire pour aider les souffrants, que ce soit dans le cadre de son pays lors d'épidémies de typhus et de choléra, ou dans le cas de la Grèce où on lui confia l'organisation de mesures sanitaires contre la peste. Son énergie débordante était canalisée non seulement dans l'action, mais également dans la réflexion. Comme écrivit Alphonse de Candolle dans une notice biographique de Louis-André Gosse :

«Son activité se répandait sur une foule d'objets, soit de pratique et d'utilité du moment, soit de théorie. Il compensait les inconvénients de cette dissémination par une disposition inverse à se laisser dominer, pendant quelque temps par chaque sujet. Chez lui les questions ne se mêlaient pas, elles se succédaient, et durant la préoccupation d'une idée il la poursuivait avec une ardeur infatigable. Ce n'est pas le système de l'application constante des hommes spéciaux à une seule branche, mais ce n'est pas non plus la diffusion des forces sur plusieurs études simultanées qui produit si rarement de bons effets. » ${ }^{4}$

Gosse avait environ 45 ans lorsqu'il écrivit son Examen médical et philosophique du système pénitentiaire, et il se préoccupa de la médecine pénitentiaire jusqu'à la fin de ses jours. Il est mort en 1873 à l'âge de 82 ans. Il importe de noter que son fils Hippolyte-Jean (1834-1901), médecin également, esprit original et polymorphe, enseigna la médecine légale à la Faculté de Droit avant la création de la Faculté de Médecine et fût nommé à la Faculté de Médecine le 4 mars 1876 pour ce même enseignement. La médecine légale à l'époque, comme actuellement dans plusieurs universités, avait comme objet d'aider la justice à découvrir la vérité. Il est intéressant de noter que dans le pays natal des Gosse, 100 ans après, l'Institut de Médecine légale a combiné les intérêts du père et du fils, offrant ses services aux justiciers aussi bien qu'aux justiciables, réunissant la médecine légale et la médecine pénitentiaire. 


\section{L'examen médical et philosophique du système pénitentiaire}

Ce qui frappe d'emblée le lecteur de cet ouvrage est le désir de l'auteur de baser son analyse du système pénitentiaire sur une connaissance scientifique du comportement de tout individu, qu'il soit en harmonie avec les lois ou pas. Gosse considère que l'homme est composé d'une organisation matérielle intimement liée à une organisation immatérielle. Après avoir admis que cette double organisation, malgré le fait qu'elle est admirablement adaptée au maintien de la vie individuelle et sociale, peut présenter des imperfections, il met l'accent sur les interactions entre les aspects physiques et mentaux :

«Si maintenant, nous envisageons l'homme dans la liaison qui existe entre son âme intelligente et le corps, nous ne pouvons méconnaître que le siège de cette liaison est ce même cerveau dont nous venons de signaler la fragilité, et que les rapports qui s'établissent entre ces deux principes sont assez intimes, pour qu'une impression ou une lésion quelconque reçue par l'un, soit immédiatement reproduite sur l'autre. Il est également évident, ou du moins très probable, que l'impression reproduite sur le cerveau est plus ou moins matérielle, qu'elle provienne de causes physiques ou morales, d'où résulte une réaction de la matière sur le principe immatériel, réaction qui persiste pendant un temps plus ou moins long, suivant l'étendue du changement matériel. $»^{5}$

L'auteur retient une série de facteurs internes et externes pouvant influencer ou dérégler le cerveau :

«des commotions accidentelles sur le crâne, la présence de corps étrangers, l'action de certaines substances alimentaires ou médicamenteuses, un trouble de la circulation cérébrale, un épanchement de sang ou de lymphe, des douleurs rhumatiques, et l'influence prédisposante des âges, des climats, des saisons, des variations atmosphériques. $\aleph^{6}$

Il considère que dans ces cas de dérèglement du cerveau et du système nerveux, la circulation sanguine est un agent actif. Nous sommes loin des théories qui attribuaient le dérèglement du comportement à une agression du cerveau "par une humeur qui avait pris naissance dans un autre lieu ». ${ }^{7}$ Après Pinel et Esquirol les nerfs et le système nerveux deviennent les concepts explicatifs fondamentaux pour l'étude du comportement. Gosse cependant comme nous verrons plus loin, malgré ce progrès conceptuel construit ses recommandations concernant le traitement physique et moral des criminels, tout comme les grands psychiatres français de l'époque, sur des idées traditionnelles issues de théories désuètes. Pour les hypocondriaques, les anorexiques, pour les suicidaires, il déclare:

«Modifiez les effets de la circulation sur le cerveau, faites cesser la congestion sanguine par un moyen quelconque, un air plus sec et plus frais, un vomitif, un purgatif, des bains de pied, une soustraction artificielle de sang, et ces mêmes individus deviennent gais, bienveillans. $»^{8}$ 
Pour les criminels il donne également des recommandations de même type. Mais Gosse ne pense pas que des causes physiques uniquement produisent des dérèglements du cerveau. Il soumet à l'examen les perturbations du cerveau causées par des excitations d'ordre mental, intellectuel ou moral. Ainsi pense-t-il que des sensations telles que la peur, la jalousie, le chagrin, les élans créateurs ou de piété, produisent sur le cerveau des impressions qui mettent en jeu une injection vasculaire, donc une excitation matérielle. Cette dernière comme dit Gosse «une fois produite, réagit à son tour sur l'agent moral et intellectuel, et cette espèce de fièvre active son développement ou son perfectionnement $"{ }^{9}$ Il est intéressant de noter que cette rétroaction se traduit en termes modernes par la notion de feedback. Gosse utilise le concept de rétroaction lorsqu'il essaie d'arriver à l'explication des comportements criminels :

«Lorsque l'action des causes morales et intellectuelles a été faible ou passagère, et qu'elle ne se répète pas, l'équilibre se rétablit, ... la stimulation vasculaire cesse, une absorption a lieu localement, et les modifications matérielles produites tendent à s'effacer insensiblement ; c'est ce qui donne lieu à l'oubli. Mais si l'action de ces causes est plus ou moins intense et durable, la stimulation vasculaire continuant, l'impression matérielle, plus persistante, se reproduit avec plus ou moins de facilité et de netteté ; et si, en outre, une même cause agit à diverses reprises sur le même individu, elle laisse des traces encore plus profondes; c'est ce qui constitue l'habitude morale. Or cette habitude est bonne ou mauvaise, suivant la nature de l'impression première. $»^{10}$

Pour l'auteur plus une habitude morale s'établit, moins l'individu peut de luimême lui résister. De plus cette habitude peut persister même si la cause morale primitive est détruite. Le principe de production de bonnes ou mauvaises habitudes morales est le même, qu'il s'agisse d'un homme noble ou vertueux ou d'un criminel.

Gosse base sa défense du système pénitentiaire sur le principe de la réversibilité des habitudes morales :

«Toutefois, quelques profondes que soient les racines qu'ont jeté les habitudes morales dans l'organisme, elles ne sont pas pour celà toujours fixes et invariables. Le même procédé qui favorise la disparition d'une impression légère, primitive, et que nous avons dit amener l'oubli, sert également à détruire les habitudes morales mauvaises ou bonnes. Ainsi, lorsque la cause morale primitive cesse d'agir, l'action des causes matérielles secondaires tend alors à diminuer, et le cerveau moins excité réagit à son tour plus faiblement sur l'âme. »11

Il base également son apologie du système pénitentiaire sur l'idée du traitement, étant admis que le criminel est souvent incapable par lui-même de régénération. Nous voyons apparaître ici un épiphénomène de la théorie de la réaction. Du fait que l'homme, en l'occurrence l'homme délinquant, a une habitude morale plus 
ou moins ancrée, il lui faut un traitement, il lui faut l'assistance de personnes compétentes, afin qu'il puisse retrouver ce qui lui fait défaut. Jean Starobinski a montré comment la réflexion qui se développe autour du concept de réaction est un moment important pour une nouvelle valorisation du rôle du médecin au $\mathrm{XIX}^{\mathrm{e}}$ siècle $^{12}$. La profession médicale ayant retrouvé son prestige, qui avait été un peu terni jusqu'à la fin du $\mathrm{XVIII}^{\mathrm{e}}$ siècle, elle peut se permettre de s'étendre, de médicaliser de nouveaux domaines, entre autres la prison.

Sur ces bases théoriques Gosse construit ses critiques du fonctionnement du système pénitentiaire en même temps que la défense des principes sur lesquels ce système repose. Postulant que toute société cherche à se protéger des hommes qui commettent des actes illégaux, Gosse affirme que pour obtenir cela, les coupables doivent en premier lieu subir une punition, afin qu'ils ne recommencent pas et qu'il y ait un effet de dissuasion sur les autres candidats à l'illégalisme. En second lieu les coupables doivent être amenés à une régénération, l'incarcération supprimant les facteurs matériels d'excitation du cerveau et produisant des excitations intellectuelles et morales qui fournissent un traitement moral proprement dit.

Gosse fait donc une distinction entre traitement physique et moral et traitement moral proprement dit. Au point de vue du traitement physique et moral il émet une série de directives, afin que la réclusion, l'isolement, le régime alimentaire, le sommeil, l'habillement, et l'habitat des détenus contribuent à leur régénération. Du point de vue du traitement moral proprement dit, il essaie de montrer comment l'éducation morale et religieuse, l'éducation intellectuelle, le travail, les habitudes d'ordre, les punitions et les récompenses peuvent contribuer à la régénération des détenus.

Nous ne considérerons pas de manière détaillée l'application au système pénitentiaire des principes de Louis-André Gosse qui en fait rejoignent le discours pénitentiaire classique au $\mathrm{XIX}^{\mathrm{e}}$ siècle, mais nous tâcherons de dégager à titre d'exemple quelques idées à propos desquelles on peut se demander dans quelle mesure les concepts scientifiques guident les applications pratiques et dans quelle mesure ils «réagissent » aux problèmes créés par l'application.

Le traitement physique et moral

Gosse pense qu'une réclusion est nécessaire pour obtenir un effet régénérateur sur les individus car il faut que les criminels soient soustraits aux «influences vicieuses extérieures ». ${ }^{13}$ Une réclusion à vie n'a pas de sens dans une optique de régénération. Pour Gosse, la réclusion «exerce une action débilitante directe et indirecte sur la constitution». Mais acceptant sa nécessité sociale ainsi que du point de vue 
du traitement il ne la met pas en question et cherche les moyens pour en diminuer les effets néfastes. Gosse a une attitude différenciée au sujet de l'isolement. Il lui attribue, lorsqu'il est pratiqué pour une courte durée, une valeur d'intimidation, de punition efficace. Il empêche la «contagion morale» entre les condamnés et empêche que la prison devienne une «école du vice». D'autre part :

«L'isolement absolu en supprimant les causes externes d'excitation, est nécessaire pour calmer l'irritation matérielle des sens, et pour ralentir la circulation abnorme du sang. En même temps il fait rentrer le coupable en lui-même, le force à réfléchir sur sa position, sur sa conduite passée, sur les agens de séduction qui l'ont entraîné, il favorise le réveil de la conscience et porte au repentir, à la résignation, aux idées religieuses. ${ }^{14}$

En revanche un isolement absolu prolongé nuit à la régénération :

"Cette habitude d'une existence morale passive et sans lutte, est, pour le condamné, une éducation fautive, puisqu'elle le rend incapable, à sa sortie, de résister aux pièges et aux séductions qui l'attendent plus tard dans la société, et que plus il aura été soumis à des privations exagérées pendant sa réclusion, plus aussi il sera tenté de se jeter dans les excès opposés lorsqu'il recouvrera sa liberté. ${ }^{15}$

Par ailleurs l'isolement absolu prolongé nuit au corps à cause du manque de mouvements, et à l'état mental qui est submergé de sentiments tristes et pénibles. Par conséquent Gosse dit :

"Il résultera une gêne dans la circulation cérébrale, le système nerveux sera faiblement excité, les fonctions de la peau languiront et le système lymphatique prédominera. ${ }^{16}$

Les détenus seront prédisposés ainsi aux maladies physiques et aux affections mentales. Si l'isolement absolu donne l'impression en apparence de simplifier l'administration et de réduire les coûts, en réalité il exige d'une part des constructions très coûteuses si on respecte certaines normes sanitaires et d'autre part en escamotant la régénération, déprécie les fonctions des employés et ne détruit pas chez le détenu les causes de ses délits.

Gosse préconise donc l'isolement relatif pour les détenus, c'est-à-dire le travail silencieux en groupe de jour et l'isolement de nuit. Ce régime est pour Gosse «plus conforme aux habitudes de l'homme en société, et à l'accomplissement de ses obligations sociales». Le silence, pour l'auteur, pendant le travail en groupe, quoique n'éliminant pas toute communication, puisqu'elle peut se faire par gestes,

«ajoute au calme matériel, en diminuant l'activité du sens de l'ouie il empêche la contagion du vice par les paroles, habitue le condamné à exercer un empire sur lui-même, favorise le travail de son intelligence... ${ }^{17}$

Les vertus du silence sont recommandées par de nombreux écrivains de l'époque. Il est intéressant de voir survivre dans le système pénitentiaire (comme d'ailleurs dans d'autres institutions totalitaires, l'école, l'usine, l'armée) sous une forme à 
peine changée, les anciennes formes disciplinaires de la pénitence et du silence. Peut-on laisser passer inaperçu que la loi du silence entre détenus permettant la communication uniquement avec les gardiens, le directeur, l'aumonier, le médecin, les visiteur charitables, ne fait qu'accentuer le poids d'un contrôle hiérarchique?

Les autres moyens qui peuvent avoir une influence directe sur le traitement physique des condamnés et indirectement sur le traitement moral sont pour Gosse le régime alimentaire, les exercices et repos du corps, le sommeil et la veille, la propreté, le chauffage et l'aération de la prison.

Il serait trop long de reprendre en détail le discours de Gosse. En résumé, partant toujours du principe qu'il faut éviter la surexcitation du système vasculaire cérébral, ainsi que du principe de la réaction réciproque du physique et du moral, il aboutit à une série de recommandations, certaines nécessitant des modifications dans la construction des pénitenciers.

Voici encore une citation caractéristique de l'époque qui montre une fois de plus les rapports thérapeutiques qui existent dans l'esprit de Gosse entre maladie mentale et criminalité.

"Qu'on force le maniaque, l'hypochondriaque, l'homme qui veut se suicider, à prendre un exercice à pied régulier, jusqu'à la fatigue musculaire, et l'état maladif du cerveau se dissipe, le calme moral se rétablit, les idées erronées qui pouvaient leur faire commettre des fautes s'évanouissent. \} ^ { 1 8 }

\section{Le traitement moral proprement dit}

Nous examinerons maintenant les réflexions de Gosse sur le traitement moral proprement dit, qui doit intervenir une fois que le traitement physique aura atténué les séquelles physiques dans le cerveau de ce qu'il appelle les mauvaises habitudes morales.

Cet aspect de la régénération comprend l'éducation morale et religieuse, l'éducation intellectuelle, le travail, les habitudes d'ordre et de propreté, les punitions et les récompenses. Les premières recommandations pour l'éducation morale sont révélatrices de la philosophie sociale de l'auteur :

«On commencera donc à lui prouver [au coupable], par des faits plutôt que par des paroles, que s'il y a injustice hors de la prison, la justice la plus stricte règne pour lui comme pour les autres dans son intérieur. On lui témoignera de l'intérêt et de la bienveillance, et on fera tout ce qu'il sera possible pour gagner sa confiance, en reconnaissant ses malheurs et évitant les points acides du souvenir de ses torts, tout en admettant qu'il y a pu avoir des causes indépendantes de sa volonté, qui l'ont conduit au vice et au crime. ${ }^{19}$ 
Gosse se trouve entre deux écoles de réaction face au crime : entre l'école classique du XVIII ${ }^{e}$ qui ne se préoccupait pas de la responsabilité individuelle mais tenait à ce que la peine administrée soit mesurée et proportionnelle au crime, et l'école positiviste de la fin du $\mathrm{XIX}^{\mathrm{e}}$ siècle dont le déterminisme nie la responsabilité individuelle ainsi que la possibilité de régénération chez les criminels. Gosse est un représentant de l'école néo-classique qui commence à tenir compte de l'irresponsabilité tout en croyant aux vertus de la punition.

Gosse qui croit à l'existence d'un sentiment inné du bien et du mal, pense que la régénération doit contribuer au réveil de la conscience. Cela fait :

«Si le condamné le désire, on s'occupera de raisonner avec lui, et en particulier, dans un langage très simple et à sa portée, sur les devoirs de l'homme envers lui-même et envers ses semblables, puis on lui démontrera que la morale n'est que son intérêt bien entendu dans ce monde et que même par égoïsme il devrait se bien conduire. $»^{20}$

Puis d'un coup de force assez paradoxal Gosse lie ses conseils basés sur une théorie hédonistique à une morale chrétienne d'abnégation de soi-même. L'appel à la pensée hédonistique serait-il l'équivalent d'un appel à la pensée du jeune enfant, et sommes-nous en présence d'une méthode psychologique bien plus tardive, concernant la régression qui permet de restructurer la personnalité ?

Une autre remarque témoigne de la subtilité du raisonnement de Gosse :

«Il ne faut jamais perdre de vue qu'on a affaire à un prisonnier, plus ou moins irrité par la contrainte qui lui est imposée, surtout dans le début de la réclusion, et que la comparaison de ce qu'il éprouve avec le sort de grands coupables qui échappent à la justice humaine, est loin d'être favorable à celle-ci. $»^{21}$

Il y a là une allusion tout-à-fait claire aux fameux chiffres noirs des criminologues modernes. Mais Gosse à nouveau ne poursuit pas cette constatation jusqu'au bout et ne s'interroge pas sur les liens de la législation et de la justice avec les intérêts des groupes dominants.

L'éducation intellectuelle est pour l'auteur importante pour une préparation professionnelle ainsi que pour son influence sur les sentiments moraux. Elle ne devrait pas être trop poussée car elle risque selon l'auteur de perturber l'harmonie du cerveau, jeter un doute sur les croyances sacrées, favoriser l'égoïsme et conduire parfois à la folie.

Le travail est pour Gosse un moyen essentiel pour la régénération des détenus. Le travail, vertu et norme, est indispensable,

«soit pour combattre les effets de l'oisiveté ou d'une activité mal dirigée, vices si fréquens chez la plupart des criminels avant leur condamnation, soit pour entretenir une activité harmonique du physique et du moral pendant leur réclusion, soit en leur donnant le goût et l'habitude du travail, et préparant ainsi les voies pour qu'ils puissent gagner honnêtement leur existence à l'expiration de la peine ${ }^{22}$ 
Comme la peine et comme le silence, le travail n'est pas sans rappeler des règles monastiques. Mais l'éthique du travail, présente déjà dans le laborare est orare, magnifiée par le protestantisme, est également une norme absolue de la société en voie d'industrialisation. Ainsi Gosse remarque-t-il «qu'on devra s'attacher aux industries de première nécessité qui offrent un gain moins précaire, et qui n'éxigent point un apprentissage trop prolongé ${ }^{23}$ Le prisonnier quittant la prison, s'il ne récidive pas, se joindra tout naturellement aux rangs du prolétariat. Par ailleurs soulager les finances de la prison est également fortement souhaité.

Gosse, comme nous l'avons déjà vu au sujet de l'éducation morale, croit à l'utilité de la punition. Mais, dit-il,

«Pour que les punitions soient véritablement utiles, elles doivent conserver le caractère imprimé par la loi au jugemens, c'est-à-dire, ne porter l'empreinte ni de la passion, ni de la brutalité, loin de gêner l'éducation du coupable, elles doivent la favoriser, enfin elles ne sauraient aggraver sa position sanitaire. $\nu^{24}$

Le dernier point est important. La santé physique et mentale du prisonnier doit être assez bonne pour qu'il puisse sentir les effets de la punition ${ }^{25}$. Peut-être faudrait-il rappeler qu'il n'y a pas très longtemps aux Etats-Unis les électrocutions étaient repoussées dans le cas où le détenu n'était pas suffisamment lucide pour comprendre la raison de sa punition.

Parmi les différents moyens d'intimidation Gosse propose l'isolement absolu (temporaire avec ou sans suppression de travail, dans des cas graves dans l'obscurité), le régime au pain et à l'eau, les exercices fatigants, la camisole de force pour les rebelles, enfin le passage d'une catégorie de condamnés à l'autre, passage vers une catégorie où la contrainte est plus sévère.

Gosse est partisan d'un système de récompense. L'homme, écrit-il,

«est, par sa nature et par ses souvenirs, plus disposé à faire des efforts pour se procurer des avantages, que pour se soustraire à des peines ${ }^{26}$

A nouveau il est question du calcul hédonique si cher à Bentham. Gosse trouve qu'il ne devrait pas y avoir d'objections concernant les récompenses qui découlent de l'idée de traitement tel que le passage d'une catégorie de prisonniers à une autre. Il s'attarde sur des sujets que ses concitoyens avaient du mal à admettre : l'allocation aux prisonniers d'une part de leurs gains et la diminution de la détention. Ces propositions préfigurent le système pénitentiaire dit Irlandais importé en Angleterre et en Irlande par un capitaine australien (Maconochie) qui préconisait le travail productif, un système de récompense, et la libération conditionnelle.

Gosse a soulevé également, rappelons que c'était en 1837, le problème des détenus aliénés. Que faut-il faire des individus qu'on envoyait en prison après qu'ils aient manifesté des symptômes d'aliénation mentale ou de ceux qui sans 
avoir manifesté aucune aliénation devenaient fous pendant la réclusion ? Il recommande la création d'un quartier criminel dans les hospices d'aliénés,

«soit pour respecter les convenances et les intérêts de la société, qui s'opposent à ce que l'on confonde les fous coupables d'un délit avec les aliénés ordinaires, soit pour pouvoir adopter des mesures de sûreté qui préviendraient toute espèce d'évasion, en admettant l'existence d'aliénations simulées ».27

De toute façon Gosse ne pense pas qu'un prisonnier voudrait de gaité de cœur «simuler une aliénation qui le soumettrait indéfiniment, à une contrainte non moins sévère que dans le pénitencier, à un traitement médical forcé plus ou moins désagréable, et à une suppression de toute espèce de récompenses ${ }^{28}{ }^{28}$

Qu'aurait-il pensé, si on lui prédisait que 130 ans après le sort des détenus souffrant de maladies mentales est encore loin d'être satisfaisant dans sa ville natale?

Gosse présente également des idées sur la construction d'une prison selon le principe de l'inviolabilité et du système panoptique, sur la séparation des diverses catégories de prisonniers selon la gravité de leurs actes, leur âge, leur sexe, les moyens de régénération qui leur sont destinés, sur le choix et les qualifications et les devoirs du personnel, du directeur, des employés subalternes, des ecclésiastiques et des médecins, sur le soutien et la surveillance des anciens détenus sortis de prison fournis par des institutions privées et étatiques ${ }^{29}$.

Les recommandations de Gosse sont le fruit d'une étude approfondie de diverses enquêtes et rapports sur les prisons américaines et européennes ainsi que des travaux devenus classiques des Crawford, Demetz et Blonet, Julius de Beaumont et de Tocqueville. Il suivait également de près ce qui se passait ailleurs en Suisse.

A Genève il a participé à la fameuse controverse entre partisans du système de Pennsylvanie et partisans du système d'Auburn. Comme nous l'avons déjà vu, Gosse était pour le système d'Auburn, c'est-à-dire le travail et les repas en commun et en silence, et l'isolement de nuit. Il était contre le modèle de Pennsylvanie, qui prescrit l'isolement absolu de jour et de nuit. Genève, dont le premier pénitencier a été ouvert en octobre 1825, avait adopté un système d'isolement relatif. Or vers les années 1840, années turbulentes du point de vue politique, on a vu naître un projet de loi sur l'organisation des prisons qui introduisait l'isolement de jour et de nuit. Le mouvement de balancier entre des mesures de répression plus ou moins grandes a toujours suivi l'agitation politique. Les défenseurs de l'isolement absolu motivaient leur choix par le fait que l'éducation pénitentiaire suivie avait été insuffisante et n'avait apporté aucune amélioration. Gosse répondit avec un livre en attaquant le projet de loi. Il admettait que tout n'allait pas pour le mieux à Genève, mais attribuait l'échec au régime de vie monotone, triste et sédentaire de la prison pénitentiaire. Il essayait de démontrer par des statistiques (assez 
douteuses il est vrai) que partout dans le monde les prisons avec isolement absolu présentaient des taux de mortalité et de maladie mentale déclanchée en prison, supérieurs à ceux des prisons à isolement relatif.

Gosse n'a pas cessé de s'intéresser au système pénitentiaire. Ainsi, en 1843, il commenta l'ouvrage du Dr Verdeil concernant la réclusion dans le canton de Vaud $^{30}$; en 1863 il adressa un programme de questions aux membres de la Société Suisse d'Utilité Publique concernant les établissements pénitentiaires ${ }^{31}$ et en 1864 il rédigea un projet d'un pénitencier industriel, agricole et éducatif accompagné par de splendides plans panoptiques de l'architecte Schæck-Jaquet ${ }^{32}$.

\section{Conclusion}

Gosse a fait une synthèse très claire et intéressante des travaux de l'époque. On ne peut dire qu'il fut un novateur, mais il a su, contrairement à la plupart des écrivains de l'époque qui se basaient sur les résultats uniquement pratiques du système pénitentiaire, présenter des réflexions dans un cadre théorique. Si sa conceptualisation peut paraître naïve actuellement, il ne faut pas oublier que les explications de type biologique qui furent présentées après lui par Lombroso et ses adeptes étaient infiniment plus grossières. Dans un certain sens, Gosse a su éviter les exagérations des théories biologiques ainsi que des théories environnementalistes.

Gosse a participé à ce mouvement de réforme de la prison qui est contemporain de la naissance de la prison, qui se poursuit de nos jours et qui défend l'idée d'une transformation, d'une correction, de la régénération du criminel comme disait Gosse. Or, nous pouvons déceler quelques problèmes dans ce mouvement de réforme.

Le premier problème concerne la relation paradoxale punition-traitement. Pour certains penseurs de l'époque, la régénération était assimilée à un traitement humanitaire. Emergeant d'une époque où torturer était courant, ces penseurs étaient placés dans une situation où il leur fallait justifier un traitement plus humanitaire, par le fait par exemple qu'il pouvait contribuer à la régénération, et partant à la diminution des récidives. Mais cette position ne pouvait qu'être à double tranchant pour les défenseurs du point de vue humanitaire. Car dans le cas où le traitement humanitaire n'apporte pas de changement considérable, ne diminue pas le nombre de récidives, ne contribue pas à l'intégration du criminel dans la société, on dira qu'il n'est pas valable et qu'il ne vaut pas la peine d'être continué. On peut cependant se demander si toutes les méthodes rééducatives ou régénératrices préconisées - amélioration de l'hygiène, travail, éducation, loisirs, formation professionnelle, respect de la dignité du détenu - peuvent avoir un autre effet que d'atténuer les effets nocifs propres à l'incarcération. 
Ces mesures tendent à humaniser le régime pénitentiaire, à réduire un peu les facteurs reconnus comme pathogènes, mais elles ne peuvent être considérées comme rééducatives ou thérapeutiques. Elles ne visent pas des hommes libres, dans leur humanité, mais l'homme détenu, c'est à dire l'homme privé de la liberté qui est partie intégrante de son humanité. Il commence à être de plus en plus reconnu que le milieu carcéral anéantit les effets de tout essai de régénération et qu'il importe de s'interroger sur la notion même de régénération, sur ce qu'elle contribue à masquer.

Cela nous mène au deuxième problème. Le mouvement de réforme de la prison au XIX ${ }^{\mathbf{e}}$ siècle, comme actuellement, a toujours reposé sur une acceptation d'une série de fonctions remplies par cette institution: fonction de pénitence et de réparation, de dissuasion, de réformation ou régénération. Cependant, à côté de ces fonctions manifestes et ouvertement reconnues il existe toute une série de fonctions latentes. En premier lieu une fonction d'épuration; il s'agit d'éloigner du système social des éléments improductifs, des individus qui par leurs idées ou leur mode de vie rappellent les imperfections de l'ordre social. En deuxième lieu une fonction de détournement; dans la prison se trouvent surreprésentées les classes pauvres pour des délits bien caractérisés qui détournent l'attention du public d'autres actes potentiellement plus dangereux mais officiellement noncriminalisés commis par des individus ou groupes plus proches du pouvoir (illégalismes financiers, trafic d'armes, spéculation immobilière, etc.). La délinquance des classes supérieures est moins visible et moins criminalisée. En troisième lieu une fonction symbolique; la réaction sociale tend à bipolariser les individus : ils sont normaux ou anormaux. Dire que ceux qui vont en prison sont des anormaux renforce ceux qui les caractérisent comme tels dans leur assurance d'être, eux, des individus normaux ${ }^{33}$.

D'autres fonctions latentes peuvent également être mentionnées, qui dépendënt des structures sociales spécifiques à chaque société. Elles sont liées aux conditions de l'emploi, à la conjoncture politique, à la recherche scientifique, à la satisfaction du besoin autoritaire, etc.

Gosse ne s'est pas posé de questions sur la relativité des normes et des valeurs et par conséquent sur la sélectivité de la réaction sociale face aux actes définis et poursuivis comme illégaux. Il ne s'est pas non plus interrogé sur le sens, la signification, l'intentionnalité que peuvent avoir certains actes délictueux. Pourtant, il n'était pas de ceux qui pensent que l'analyse biologique du comportement peut expliquer complètement ce comportement; il attribuait une grande importance au milieu. Gosse était probablement conscient qu'il existe une rupture épistémologique cruciale entre les sciences physiques et les sciences psycho-sociales, due au 
fait que la relation entre un comportement et des déterminismes biologiques ou sociaux est médiatisée par la pensée et l'intention humaines. Mais il utilisait un concept métaphysique pour cette médiation: la conscience, qu'il considérait comme un principe divin. Est-ce pour cela qu'il n'a pas osé pousser plus loin l'analyse scientifique de ce principe de médiation ? Le rôle du scientifique est-il d'accepter sans sourcillier la définition sociale ou religieuse de ce qui est bien et ce qui est mal ou plutôt d'étudier en vertu de quels mécanismes ces définitions, aux implications graves, sont attribuées?

Gosse vivait dans un milieu où la science était très estimée. Une science qui postulait un ordre dans l'univers, dans la nature comme dans la société. Ses idées sur la criminalité et sur le système pénitentiaire découlaient de ce postulat; il était cohérent avec lui-même. Mais la cohérence est-elle indispensable ?

\section{Bibliographie et notes}

${ }^{1}$ Cf. Radinowicz, Leon, Ideology and Crime: A Study of Crime in its Social and Historical Context, London (Heinemann) 1966, p. 38-39.

${ }^{2}$ Gosse, L.-A., Examen médical et philosophique du système pénitentiaire, Genève (Lador et Ramboz) 1837.

${ }^{3}$ Reverdin, F., Notes biographiques sur les chimistes ayant pris part à la fondation de la Société Helvétique des Sciences Naturelles, Genève 1916, p. 5.

${ }^{4}$ De Candolle, A., Louis-André Gosse. Mémoires de la Société de Physique et d'Histoire Naturelle de Genève, 1873, p. 462-464.

${ }^{5}$ Gosse, L.A., op. cit., p. 8.

6 ibid., p. 9.

7 cf. Starobinski, J., Histoire du traitement de la mélancolie des origines à 1900. Acta psychosomatica, Bâle (J.R.Geigy) 1960, p. 49.

${ }^{8}$ Gosse, L.A., op. cit., p. 11.

9 ibid., p. 13.

10 ibid., p. 14.

11 ibid., p. 16.

12 Starobinski, J., Réaction, le mot et ses usages, in : Confrontations psychiatriques, 1974, $\mathrm{N}^{\mathrm{o}} 12$, p. 19-42.

${ }^{13}$ Gosse, L.A., op. cit., p. 25.

14 ibid., p. 28.

15 ibid., p. 29.

16 ibid., p. 30.

17 ibid., p. 32.

18 ibid., p. 37. Gosse poursuit : «Qu'on fatigue l'individu dont les penchans instinctifs sont très développés ou qui a contracté des habitudes vicieuses, et le cervelet étant moins excité, ces penchans se calment et ces habitudes vicieuses disparaissent.» ibid., p. 37. Gosse fait ici allusion à la masturbation.

19 ibid., p. 46. 
20 ibid., p. 46.

21 ibid., p. 48 , n. 1.

22 ibid., p. 53.

23 ibid., p. 56.

24 ibid., p. 58.

${ }^{25}$ Pour Charles Coindet, contemporain de Gosse, cela est encore plus clair: «lorsque le prisonnier est rebelle, il faut pouvoir le dompter, en employant les moyens pénaux propres au système d'intimidation, et pour cela il faut que sa santé lui permette d'en subir l'épreuve». Dans Mémoire sur l'hygiène des condamnés détenus, Paris (Baillière) 1838, p. 82.

${ }^{26}$ Gosse, L.-A., op. cit., p. 63.

27 ibid., p. 70.

${ }^{28}$ ibid., p. 70.

${ }^{29} \mathrm{Si}$ nous reprenons les sept maximes universelles de la bonne «condition pénitentiaire» relevées par M. Foucault comme étant présentes depuis le premier tiers du $\mathrm{XIX}^{\mathrm{e}}$ siècle dans les travaux sur la réforme pénitentiaire, nous nous aperçevons que Gosse les a tous traités : 1) le principe de la correction, 2) le principe de la classification, 3) le principe de la modulation des peines, 4) le principe du travail, 5) le principe de l'éducation pénitentiaire, 6) le principe du contrôle technique de la détention, 7) le principe des institutions annexes. Cf. Foucault, M., Surveiller et punir, Paris (Gallimard) 1975, p. 274-275.

${ }^{30}$ Gosse, L.A., Analyse raisonnée de l'ouvrage du Dr.Verdeil intitulé de la réclusion dans le canton de Vaud, Genève (F. Ramboz) 1843.

${ }^{31}$ Gosse, L.A., Enquête sur les prisons, Genève 1863.

${ }^{32}$ Gosse, L.A., Projet d'un pénitencier industriel, agricole et éducatif pour les prisonniers adultes du sexe masculin, Genève (Fick) 1864.

${ }^{33}$ Le problème de l'étiquetage psychopathologique et pseudoscientifique a été mis en évidence bien avant les sociologues théoriciens du «labeling» par Ackerknecht, E.H., Psychopathology, Primitive Medicine and Primitive Culture, in: Bull. Hist. Med. 12 (1943), p. 30-67.

\section{Summary}

Louis-André Gosse (1791-1873) belonging to a Genevan family of scientists and doctors was a keen observer of the penitentiary system of the XIXth century. In contrast to most of his contemporaries who were practically oriented, he tried to integrate his point of view and recommendations regarding prisons into a theoretical framework concerning the origins of criminal behavior. His analysis of biological factors does not fall into the extremes of biological determinism which maintains that physical factors are the sole and chief causes of crime. L.-A. Gosse made some pertinent remarks as regards the influence of institutional and social factors in the explanation of crime. However, he took social reaction to crime for granted. Given his social and scientific background his was a logical position.

Madame Cléopâtre Montandon, Ph. D.

Institut de médecine légale de l'Université

8, passage de la Radio

CH-1205 Genève 